\title{
Support for active surveillance in patients aged $\geq 75$ years
}

The survival benefit associated with nephrectomy does not justify its use in patients aged $\geq 75$ years, according to new data from Maxine Sun and colleagues, recently published in European Urology.

Reports from the recent EAU congress in Milan suggest that active surveillance should not be advocated for any patient with a small renal mass, whereas AUA and EAU guidelines state that observation is an acceptable option for elderly patients with comorbidities. Amid this confusion, Sun and her team report their evaluation of the trade-offs between surgery and active surveillance in a population-based cohort.

Researchers used a competing-risk model to assess cancer-specific mortality (CSM) against the risk of other-cause mortality (OCM), which is understandably higher in older patients. "Consequently, our approach provides a more accurate estimation of CSM risk in our patient sample than Cox-based assessment," explains Andreas Becker, coauthor of the study.
4 ...surgical-induced renal dysfunction ... might undermine the curative intent of nephrectomy... 77

Nephrectomy (whether partial or radical) was significantly associated with a lower risk of CSM than active surveillance in the total cohort of 10,595 patients with tumours $\leq 7 \mathrm{~cm}$, aged $\geq 66$ years. However, this benefit was lost when the population was restricted to those aged $\geq 75$ years $(n=4,830)$. For these patients, no significant CSM benefit was found for partial nephrectomy compared with nonsurgical management (HR 0.48, 95\% CI $0.20-1.14 ; P=0.1$ ) or radical surgery compared with nonsurgical management (HR 0.57, 95\% CI 0.32-1.03; $P=0.01$ ).

OCM rates were high for patients aged $\geq 75$ years who underwent nephrectomy (46\% and 38\% for radical and partial nephrectomy, respectively). "We hypothesize that surgical-induced renal dysfunction and cardiovascularrelated events might undermine the curative intent of nephrectomy in such patients," Becker suggests.

Unfortunately, these findings do not provide a simple solution to the treatment dilemma. "It is well known that biological age can vary substantially from chronological age," says Becker. "Further research should focus on the more accurate selection of patients for surgical excision, taking into account both the risk of disease progression and competing risks."

Sarah Payton

Original article Sun, M. et al. Management of localized kidney cancer: calculating cancer-specific mortality and competing risks of death for surgery and nonsurgical management. Eur. Urol. doi:10.1016/ j.eururo.2013.03.034 